\title{
Dynamic Lumbopelvic Stabilization Exercise Protocol for the Treatment of Stress Urinary Incontinence in Women
}

\author{
Nathalia de Souza Abreu Freire ${ }^{1 *}$, José Murilo Bastos Netto ${ }^{2}$ and André Avarese Figueiredo ${ }^{2}$ \\ ${ }^{1}$ Medical and Health Sciences College of Juiz de Fora, Hospital and Maternity Terezinha de Jesus, Salgado de Oliveira University, Brazil \\ ${ }^{2}$ Medicine College and Graduate Program in Brazilian Health of Federal University of Juiz de Fora, Brazil
}

Submission: October 18, 2017; Published: February 27, 2018

*Corresponding author: Nathalia de Souza Abreu Freire, Medical and Health Sciences College of Juiz de Fora, Hospital and Maternity Terezinha de Jesus, Salgado de Oliveira University, Juiz de Fora campus, Laguna Street, 11/101 -Jardim Glória -CEP 36015-230 -Juiz de Fora/MG, Brazil, Tel/Fax: 32-2101-5000;Email: nathyfst@gmail.com

Abstract

Objectives: To teste a lumbopelvic stabilization exercise protocol and to evaluate its effectiveness in continence and quality of life in women with urinary incontinence and to identify the perception of improvement after treatment.

Method: The treatment protocol was divided into four stages according to the degree of complexity. The exercises were performed in the dorsal decubitus position, ortostástica, sitting and quadruped with maximum repetitions without compensation. The Incontinence Severity Index found severity of incontinence and King's Health Questionnaire impact on quality of life. The Patient Global Impression of Improvement Questionnaire assessed the perception of improvement.

Results: Ten women with a mean age of $49 \pm 8.9$ years old (extremes 34 and 61 years old) have integrated the sample, eight of them completed the treatment. All had "severe" or "moderate" loss at baseline as Incontinence Severity Index. After the intervention, seven rated their losses in "mild" or "moderate" and ninety days after the end of treatment, all losses classified as "mild" or "moderate". The negative impact of incontinence on quality of life according to the King's Health Questionnaire was reduced over time, especially when comparing the initial and final evaluations. Regarding the Patient Global Impression of Improvement Questionnaire, seven women reported being "much better" or "better" comparing to the beginning of treatment and a "slightly better".

Conclusion: The stabilization exercise protocol proposed was effective for continence outcomes and quality of life and there was a favorable impact on the perception of improvement.

Keywords: Urinary incontinence; Physical therapy; Conservative treatment; Biomechanical

\section{Introduction}

The pelvic floor muscles act in synergism with the muscles of the lumbopelvic region to stabilize the lumbar spine and allow urinary continence [1,2]. Postural changes may favor incontinence as well as the correction of these changes for lumbopelvic stabilization exercises can treat urinary losses $[3,4]$. Still the gold standard physiotherapy treatment for urinary incontinence is based exclusively on exercises to strengthen the pelvic floor muscles, which tend to have reduced over time effectiveness [5], forcing patients to resort periodically to professional assistance.

Although studies proving the effectiveness of exercises for lumbopelvic stabilization treatment of patients with urinary incontinence are still incipient in the literature, the results are quite encouraging [3,6-8]. There isn't, however, methodological similarity, which hinders accurate conclusions about the effectiveness of this approach [9].

Knowing that stabilization exercises are able to change the default drive motor [1-3,9], we believe that they can bring more lasting clinical results for patients with urinary incontinence. For this reason, we plan in our service a randomized clinical trial. Thus, the first step was to evaluate the effectiveness of the lumbopelvic stabilization exercise protocol. We present the protocol and the most significant results.

\section{Materials and Methods}

This study was conducted in accordance with ethical guidelines and the Declaration of Helsinki of 1975, revised in 
1983, after approval from the Federal University of Juiz de Fora Research Ethics Committee. To test the experimental protocol of a randomized clinical trial were eligible literate women aged greater than 18 years with complaints of urinary leakage on effort. After signing the Informed Consent, due to the difficulty in obtaining women that met the established inclusion criteria, ten women with a mean age of $49 \pm 8.9$ years old (extremes 34 and 61 years old) were assessed by the provocation of cough test to confirm the presence of incontinence. The Incontinence Severity Index was chosen to verify the severity of incontinence and King's Health Questionnaire for identifying the impact of incontinence on quality of life. All instruments were administered before and at the end of 90 days after the intervention. The perception of improvement was evaluated according to the Patient Global Impression of Improvement Questionnaire after treatment and 90 days later.

Table 1: Dynamic lumbopelvic stabilization exercise protocol for the treatment of stress urinary incontinence in women.
It should be clarified that the 90-day follow-up time is justified by the need to identify if the results achieved in the post-intervention evaluation are maintained over time and may justify the follow-up losses of the sample. In the dynamic lumbopelvic stabilization exercise protocol proposed for each stage of treatment were repeated until the correct biomechanical pattern was achieved. From the right move, the number of repetitions varied between 4 and 8 movements per year. The criterion for switching was the spontaneous report of fatigue or observing inaccurate motion by the physiotherapist. All exercises were performed in the dorsal decubitus position, ortostástica, sitting and quadruped with maximum repetitions without compensation. The sessions were held individually twice a week over five weeks, totaling 10 sessions lasting approximately 30 minutes each.

\begin{tabular}{|c|c|}
\hline $\begin{array}{l}\text { Stage I ( } 1^{\text {st }} \text { and } 2^{\text {nd }} \\
\text { sessions) }\end{array}$ & $\begin{array}{l}\text { General guideline: perform isolated muscle contractions in each position (supine, sitting, quadruped and standing); } \\
\text { perform } 10 \text { repetitions of } 10 \text { seconds; should guide the patient to incorporate the co-contraction of the lower trunk } \\
\text { muscles(or internal drive) in carrying out all the exercises ("expiration = motion") as well as in daily life and working } \\
\text { activities. } \\
\text { Exercise 1. predominant muscles of the pelvic diaphragm contraction: telling the location of the muscles and asking } \\
\text { the patient to decrease the distance between the coccyx and the pubic symphysis ("suspend the vagina" or "hold } \\
\text { pee"). } \\
\text { Exercise 2. Predominant contraction of transversus abdominis: feel the contraction of the transversus abdominis } \\
\text { by palpation below the anterior superior iliac spines or by palpation of the apex of the sacrum and its perception of } \\
\text { bending motion; maintaining the buttock relexed. } \\
\text { Exercise 3. Contraction of the lower trunk muscles: associate exercises } 1 \text { and } 2 \text { keeping the lumbar spine and pelvis } \\
\text { stable. }\end{array}$ \\
\hline $\begin{array}{l}\text { Stage II }\left(3^{\text {rd }} \text { and }\right. \\
\left.4^{\text {th }} \text { sessions }\right)\end{array}$ & $\begin{array}{l}\text { General guideline: to perform movement of the upper and lower limbs associated with activation of the indoor unit; } \\
\text { hold } 3 \text { to } 8 \text { repetitions without compensations. } \\
\text { Exercise 1. Patient in supine, hips and knees flexed, feet flat- Elevação da pelve mantendo-a em posição neutra } \\
\text { - Flexion (about } 45^{\text {o }} \text { ) alternating the hip keeping the knee extended } \\
\text { - Flexion of the supported knees and feet, allowing alternating hip rotation (about } 20^{\circ} \text { ) } \\
\text { Exercise 2. Patient sitting on her sitting bones in backless chair, feet on the floor } \\
\text { - Training to sit and stand up } \\
\text { - Alternating knee extension (no load) } \\
\text { - Anterior tilt of the trunk } \\
\text { - Alternate flexion of upper limbs (no load) } \\
\text { - Simultaneous flexion of the upper limbs (no load) } \\
\text { Exercise 3. Pacient in fours } \\
\text { - Move the pelvis posteriorly and return to starting position (maximum amplitude of } 30^{\circ} \text { ) } \\
\text { Exercise 4. Patient in standing position } \\
\text { - Transference of weight to the lower limbs (lateral-lateral, anteroposterior) } \\
\text { - Mini squats with spine and pelvis against the wall and maintaining neutral pelvis } \\
\text { - To go up and and down the steps while maintaining neutral pelvis }\end{array}$ \\
\hline
\end{tabular}




\begin{tabular}{|c|c|}
\hline $\begin{array}{l}\text { Stage III }\left(5^{\text {th }} \text { to } 7^{\text {th }}\right. \\
\text { sessions) }\end{array}$ & $\begin{array}{l}\text { General guideline: Keep stabilization (stable column) during the controlled lumbar and pelvic movement on uneven } \\
\text { surfaces; hold } 3 \text { to } 8 \text { repetitions without compensations } \\
\text { Exercise 1. Patient in prone on ball with diameter compatible with her biotype. } \\
\text { - Alternating extension of upper limbs with supported knees on the ground } \\
\text { - Simultaneous extension of upper limbs with supported knees on the ground } \\
\text { - Alternating extension of the hip with her hands resting on the floor. } \\
\text { Exercise 2. Patient sitting on her sitting bones in a ball with diameter compatible with the biotype. } \\
\text { - Move the pelvis in the anteroposterior direction } \\
\text { - Move the pelvis towards laterolateral } \\
\text { - Move the pelvis in the craniocaudal } \\
\text { - Extension of alternating knees (with load) } \\
\text { - Forward tilt of the trunk } \\
\text { - Alternating flexion of the upper limbs (with load) } \\
\text { - Simultaneous flexion of the upper limbs (with load) } \\
\text { Exercise 3. Patient in standing position } \\
\text { - Forward tilt of the trunk (about 30) on jump } \\
\text { - Mini squat jump without support maintaining neutral pelvis } \\
\text { - Squat jump on simulating catching object from the floor }\end{array}$ \\
\hline $\begin{array}{c}\text { Stage IV } \\
\left(8^{\text {th }} \text { and } 10^{\text {th }}\right. \\
\text { sessions })\end{array}$ & $\begin{array}{l}\text { General guideline: Perform movements in higher speed (in the ability of each patient); hold } 3 \text { to } 8 \text { repetitions without } \\
\text { compensations } \\
\text { Exercises from stage II } \\
\text { Exercises from stage III } \\
\text { Walking exercises in stationary treadmill }\end{array}$ \\
\hline
\end{tabular}

\section{Results}

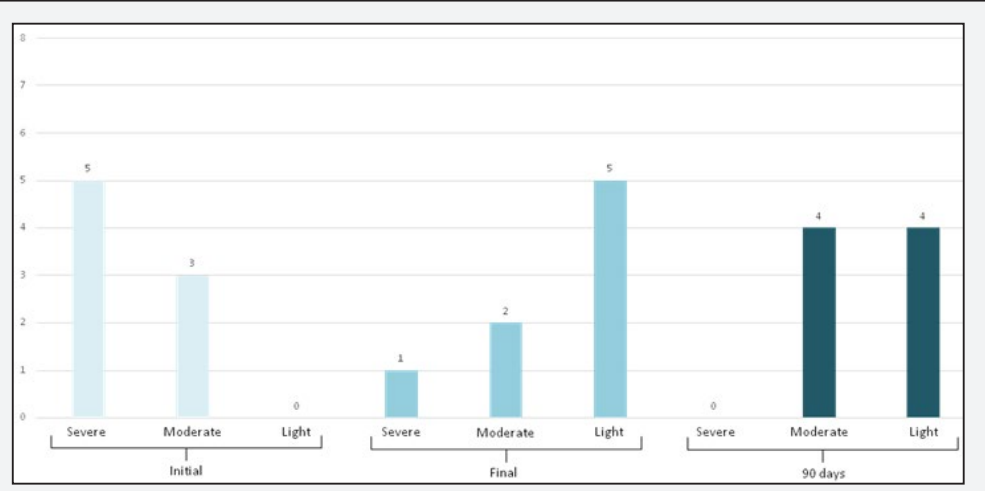

Figure 1: Severity of urine loss under incontinence severity index starting, at the end and 90 days after the end of treatment.

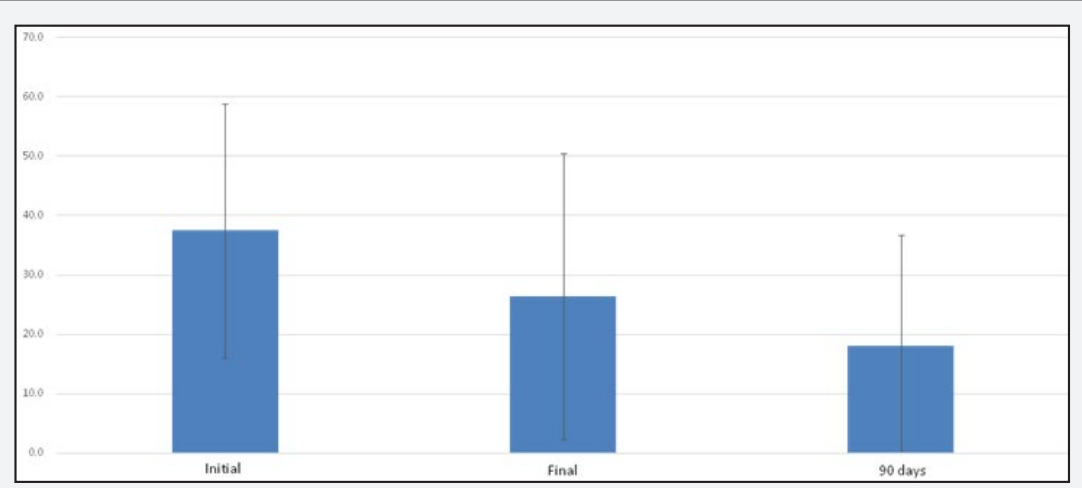

Figure 2: Impact of incontinence according to the king's health questionnaire initial, final and 90 days after the end of treatment. 
Eight women completed the treatment. The severity of incontinence before the intervention was "severe" or "moderate" for all of them. After the intervention according to Incontinence Severity Index seven women rated their losses from "mild" or "moderate" and 90 days after treatment, all reported mild or moderate losses (Figure 1). The negative impact of losses in quality of life according to the King's Health Questionnaire was reduced over time, especially when comparing to the initial and final evaluations (Figure 2). Regarding the Patient Global Impression of Improvement Questionnaire, both the final as 90 days later, seven women reported being "much better" or "better" in relation to beginning of treatment and a "slightly better".

\section{Discussion}

Urinary incontinence is a common condition among women, including those physically active. It favors emotional damage and social isolation that culminate in the decrease of quality of life. In this sense, the treatment is necessary and has as the central objective to reduce the waste and to improve the quality of life.

In the present study, the assessments conducted immediately after and 90 days after the end of the treatment showed a reduction of the frequency and volume of urinary output, showing the effectiveness of the lumbopelvic stabilizing protocol proposed when it comes to mitigating urinary losses not only in the post intervention, but also over time. It is likely that this result is related to the ability of stabilization exercises to change motor control $[1-3,9]$. Thus, even after treatment discontinuation muscle activation learned during the intervention remains, mitigating the chances of recurrence or worsening of losses. In our study, there was even a woman who experienced a reduction in severity of incontinence after the end of the intervention. On the other hand, the treatment of urinary incontinence based on exercises for the pelvic floor muscles, considered level of evidence " $\mathrm{A}$ ", tends to be favorable in the short term and disappointing after stopping treatment because quite frequently the women return episodes of loss over time [5], situation easily observed in the clinical setting and proven in literature [3].

The reduction of the frequency and volume of the losses reflected in the lower impact of quality of life, especially in the comparison of the initial and the final assessments, which can be explained by the natural tendency of greater improvement during the intervention. However, when stopping the treatment perceives restricted decline, suggesting that the requisite quality of life benefits of the motor control can extend after the end of treatment period. The positive impact of kinesiotherapeutic treatment on quality of life of women with urinary incontinence is well established in the literature. But evidence of the impact of dynamic stabilization in quality of life are restricted [3], as well as the static stabilization [6].

Hung et al. [3] in a study comparing exercise protocol of coordinated activation of the stabilizing muscles of the lower trunk versus home exercises self monitored for pelvic floor muscles, which reported outcomes in quality of life and severity of losses the results were more significant in the group submitted to exercises for the lower trunk. These authors, however, performed intervention of four months, two evaluations (pre and post treatment) and other instruments used to measure outcomes. Fozzatti et al. [6] submitted 26 women with urinary incontinence based on the postures of Global Postural Reeducation and found cure or reduce of losses in 22 of them with positive impact on all of the variables questions evaluated, including quality of life measured by the King's Health Questinnaire treatment.

Though important, the studies that concerned about identifying the perception of improvement in patients submitted to certain treatment are limited, especially in physiotherapy for urinary incontinence. This outcome allows the researcher to identify the impact of intervention in diverse and complementary perspective to the statistics, since no significant results can be considered statistically relevant to the patient. In this regard, in order to identify the perception of improvement of the women involved in the study was used instrument that showed improvement in positive perceived immediately after treatment and maintaining this insight 90 days. Increased perceived after static stabilization improvement was also reported by Fozzatti et al. [6], however the instrument of measurement was the Questionnaire Overall Impression of Improvement. No studies were found for dynamic stabilization treatment for urinary incontinence that evaluated this outcome. For this reason the present article presents data referring to the treatment protocol, which was later used in an experimental study.

\section{Conclusion}

We conclude that the stabilization exercise protocol proposed had effective outcomes for continence and quality of life in women with urinary incontinence during the study period and there was a favorable impact on the perception of improvement. Given the promising initial results and the scarce literature on the effectiveness of exercise for lumbopelvic stabilization treatment of women with urinary incontinence, we proceed with a randomized study comparing the protocol used to drive the exercises of the pelvic floor muscles, considered the gold standard in physical therapy conservative for stress urinary incontinence.

\section{Contributions of Authors}

The authors Nathalia Abreu de Souza, José Murilo Bastos Neto and André Figueiredo Avarese declare no conflict of interest and confirm participation and public responsability in the present work. Nathalia de Souza Abreu undertook the data collection, analysis and interpretation of results and writing of the manuscript. José Murilo Bastos Neto participated in the organization, analysis and interpretation of results, as well as André Avarese Figueiredo, responsible for guiding this work. 


\section{References}

1. Sapsford RR, Hodges PW, Smith MD (2010) Systematic review: Abdominal or pelvic floor muscle training. Neurourol Urodyn 29(5): 800-801.

2. Crommert AEME, Thorstensson A (2009) Trunk muscle reactions to sudden unexpected and expected perturbations in the absence of upright postural demand. Experimental Brain Research 196(3): 385392.

3. Hung HC, Hsiao SM, Chih SY, Lin HH, Tsauo JY (2010) An alternative intervention for urinary incontinence: retraining diaphragmatic, deep abdominal and pelvic floor muscle coordinated function. Man Ther 15(3): 273-279.

4. Sapsford RR (2004) Rehabilitation of pelvic floor muscles utilizing trunk stabilization. Man Ther 9(1): 3-12.

5. Dumoulin C, Hay Smith J (2008) Pelvic floor muscle training versus no treatment for urinary incontinence in women. A Cochrane systematic review. Eur J Phys Rehabil Med 44(1): 47-63.
6. Fozzatti MCM, Palma P, Herrmann V, Dambros M (2008) Impacto da reeducação postural global no tratamento da incontinência urinária de esforço feminina. Revista da Associação Medica Brasileira 54(1): 1722.

7. Painter EE, Ogle MD, Theyhen DS (2007) Lumbopelvic dysfunction and stress urinary incontinence: a case report applying rehabilitative ultrasound imaging. J Orthop Sports Phys Ther 37(8): 499-504.

8. Matheus LM, Mazzari CF, Mesquita RA, Oliveira J (2006) Influência dos exercícios perineais e dos cones vaginais, associados à correção postural, no tratamento da incontinência urinária feminina. Revista Brasileira de Fisioterapia 10(4): 387-392.

9. Bø K, Mørkved S, Frawley H, Sherburn M (2009) Evidence for benefit of transversus abdominis training alone or in combination with pelvic floor muscle training to treat female urinary incontinence: A systematic review. Neurourol Urodyn 28(5): 368-373. 